Ensino, Saúde e Ambiente - V 7 (1), Edição Especial, maio de 2014

\title{
PARASITOSES INTESTINAIS DO MUNICÍPIO DE NOVA ESPERANÇA DO PIRIÁ: CONHECER PARA EVITAR
}

\section{INTESTINAL PARASITES IN THE CITY OF "NOVA ESPERANÇA DO PIRIÁ": KNOW TO AVOID}

\author{
Maria Marcilene Alves da Silva ${ }^{1}$, Rosilene Mota de Lima ${ }^{2}$, Sandra Nazaré Dias Bastos ${ }^{3}$ \\ ${ }^{1}$ Secretaria Municipal de Educação de Nova Esperança do Piriá-PA/ marcilenealves1911@hotmail.com \\ ${ }^{2}$ Secretaria Municipal de Educação de Capitão Poço-PA \\ ${ }^{3}$ Universidade Federal do Pará (UFPA)/IECOS/e-mail: sbastos@,ufpa.br
}

\section{RESUMO}

O objetivo desse trabalho foi investigar as parasitoses intestinais mais frequentes em Nova Esperança do Piriá e promover atividades educativas na escola visando alertar a população local e minimizar o problema no município. A partir dos registros disponibilizados pelo laboratório de análises clínicas do hospital municipal, verificamos que a faixa etária com maior frequência de indivíduos parasitados foi a de crianças entre 1 e 6 anos de idade (51\%, n= 89). Ascaris lumbricoides e Giardia lamblia foram os parasitas mais frequentes. Esses dados foram discutidos por professores e alunos do $6^{\circ}$ ano e culminaram com a exposição do problema na feira de ciências da escola, evento que reúne além da comunidade escolar, pessoas da comunidade extraescolar.

Palavras-chave: Enteroparasitoses, Parasitoses, Educação e Saúde

\begin{abstract}
The aim of this study was to investigate the most common intestinal parasites in Nova Esperança do Piriá and promote educational activities in school to warn the local population and minimize the problem in the city. We found out from the records made available by the clinical analysis laboratory of the municipal hospital that the age group with the highest frequency of infected individuals was children between 1 and 6 years old $(51 \%, \mathrm{n}=89)$. Ascaris lumbricoides and Giardia lamblia were the most common parasites. These data were discussed by teachers and students of the 6th year and culminated with the exposure problem in the school science fair, an event that brings together the school community and people from outside the school community.
\end{abstract}

Key words: Enteroparositosis; Parasites; Education and Health 
Ensino, Saúde e Ambiente - V 7 (1), Edição Especial, maio de 2014

\section{INTRODUÇÃO}

As infecções por parasitas intestinais representam um problema de saúde pública mundial de difícil solução. Têm alta prevalência em nosso país, principalmente na população pobre e em crianças devido às precárias condições de saneamento básico, habitação e educação (MELO, et al. 2004). Tais doenças são pouco discutidas na área da educação, apesar disso e, tentando caminhar na contramão desse comportamento, acreditamos que através da parceria entre a escola e saúde é possível sensibilizar a sociedade evitando que crianças, adolescentes, jovens ou adultos se tornem potenciais hospedeiros de enteroparasitas.

Normalmente as crianças constituem a parcela mais atingida da população e a incidência de parasitoses nessa fase reflete diretamente sobre o rendimento escolar, promovendo a incapacitação física e intelectual dos indivíduos parasitados (MACEDO, 2005).

Condições precárias de higiene, ausência de informações sobre medidas preventivas são fatores que contribuem para que as populações menos favorecidas e em especial as crianças se tornarem público alvo para proliferação dos parasitas intestinais. Nova Esperança do Piriá não dispõe de saneamento básico, água tratada e coleta de lixo por isso, se apresenta como local propício para disseminação de parasitoses intestinais. Considerando essa problemática, pretendemos discutir propostas educativas que venham a ser traduzidas em ações inicialmente pelas crianças e posteriormente pela população como um todo.

Partimos do princípio de que a educação associada à saúde rende resultados positivos e satisfatórios, dessa forma, nossa pesquisa pretende contribuir para evidenciar o papel de cada um na profilaxia e controle das doenças ocasionadas por protozoários e helmintos, possibilitando compreender as relações entre saúde e qualidade de vida.

Como professoras não podemos nos furtar a essa discussão, uma vez que percebemos que em Nova Esperança do Piriá os casos de enteroparasitoses são muito frequentes. Decidimos discutir essa temática tentando aproximar os temas Educação e Saúde, com o objetivo de promover uma ação integrada visando minimizar a incidência dessas doenças. A opção pelo tema como objeto de estudo foi no intuito de identificar quais as enteroparasitoses mais frequentes no município e a partir de então propor e executar ações educativas que venham informar a população sobre atitudes simples que cada um possa desenvolver, para minimizar o problema. 


\section{Ensino, Saúde e Ambiente - V 7 (1), Edição Especial, maio de 2014}

Segundo Senna-Nunes (2001), as parasitoses intestinais representam uma boa estratégia de aprendizado, e a escola pode ser um centro de debates e informações para a população periférica, envolvendo as crianças como agentes multiplicadores da saúde.

Dessa forma, os objetivos desse trabalho são: investigar as parasitoses intestinais mais frequentes no município de Nova Esperança do Piriá, associar a incidência das ocorrências por faixa etária e promover nas escolas atividades educativas que abordem os cuidados básicos e hábitos de higiene, bem como métodos preventivos.

\section{MATERIAL E MÉTODOS}

O município de Nova Esperança do Piriá está localizado na região nordeste do estado do Pará, a $315 \mathrm{~km}$ da capital Belém. Com população de 20.150 habitantes e território aproximado de 2.192,4 km², sua principal via de acesso é a rodovia PA 124. Atualmente a cidade dispõe de 52 unidades de ensino entre creches e escolas de ensino fundamental e médio (este ofertado apenas em uma escola estadual na sede do município e em duas agrovilas, sempre na modalidade modular). $\mathrm{O}$ atendimento de saúde conta com um médico que atende no hospital e no posto de saúde que estão localizados na sede do município, nas demais localidades os postos de saúde contam com enfermeiras e/ou técnicos de enfermagem. No que se refere ao trabalho da Vigilância Sanitária o município dispõe de 08 agentes de endemias, e 70 agentes de Saúde, que atendem todo o município dentre zona urbana e rural (IBGE, 2010).

Para fazer o levantamento das principais enteroparasitoses que acometem a população de Nova Esperança do Piriá, foram verificados os registros dos resultados dos exames realizados no período de seis meses no laboratório do Hospital e Maternidade do Povo do município. Os casos positivos foram organizados em uma planilha e depois transformados em gráficos e tabelas para melhor descrição e interpretação dos resultados. A partir dos resultados foram elaboradas atividades para serem desenvolvidas na escola com a participação de professores e alunos do $6^{\circ}$ ano do Ensino Fundamental.

\section{RESULTADOS E DISCUSSÃO}

Para verificar os enteroparasitas mais frequentes em Nova Esperança do Piriá foram analisados os resultados dos exames realizados no período compreendido entre julho e dezembro de 2012 no Hospital e Maternidade do Povo do município. Esses exames foram realizados em pacientes vindos de diversos bairros e agrovilas, e para Edição Especial com os melhores trabalhos apresentados no IV ENECiências: UFF - 13 a 16 de maio de 2014. 
efeitos desse estudo foram separados apenas aqueles que apresentaram resultado positivo para organismos patogênicos, em um total de 218 diagnósticos assim distribuídos: 142 na faixa etária de 0 a 12 anos, 15 para a faixa etária de 13 a 25 anos e 61 exames para a faixa etária acima de 50 anos.

Os registros foram separados nas seguintes categorias: crianças, jovens e adultos. Verificamos que a categoria mais acometida por parasitas corresponde às crianças (65\%), seguida dos adultos (28\%). Os jovens tiveram frequência de $7 \%$.

Na prevalência de casos por faixa etária, considerando a categoria de crianças, a mais acometida é a que reúne os indivíduos de um a três anos de idade (33\%), seguida da faixa que engloba os de quatro a seis anos (30\%) e os de sete a nove anos $(24 \%)$. Entre os adultos, verifica-se que a maior prevalência corresponde à faixa etária entre 26 e 50 anos chegando a $44 \%$ dos casos analisados (Tabela 1 ).

Tabela 1 - Frequência de casos positivos por faixa etária dentro dos grupos crianças, adolescentes e adultos de julho a dezembro de 2012 no Município de Nova Esperança do Piriá-Pará

\begin{tabular}{|c|c|c|c|}
\hline Categoria & Faixa Etária & $\mathbf{N}$ & $\%$ \\
\hline \multirow{6}{*}{ Crianças } & $>1$ não & 4 & 3 \\
\hline & 1 a 3 anos & 47 & 33 \\
\hline & 4 a 6 aos & 42 & 30 \\
\hline & 7 a 9 anos & 34 & 24 \\
\hline & 10 a 12 anos & 15 & 11 \\
\hline & TOTAL & 142 & $100 \%$ \\
\hline Jovens & 13 a 17 anos & 15 & \\
\hline \multirow{4}{*}{ Adultos } & 18 a 25 anos & 23 & 38 \\
\hline & 26 a 50 anos & 27 & 44 \\
\hline & $<50$ anos & 11 & 18 \\
\hline & TOTAL & 61 & 100 \\
\hline & TOTAL DE CASOS & 218 & \\
\hline
\end{tabular}

Verificamos que $86 \%$ das ocorrências foi positiva para protozoários. Os parasitas mais frequentes foram Giardia lamblia (88\%) e Ascaris lumbricoides (77\%). Os demais parasitas aparecem em baixa frequência: Entamoeba histolytica (12\%), Strongyloides stercoralis e ancilostomídeos (ambos com 10\%) e Trichuris trichiura $(3 \%)$.

Verificamos que a frequência de parasitismo aumenta dentro das faixas de idade consideradas na categoria criança e decresce com o aumento da idade e a passagem para a adolescência e a idade adulta. A faixa etária com maior frequência de indivíduos parasitados foi a de crianças entre 1 e 6 anos de idade $(51 \%, n=89)$, tanto 
para os protozoários, quanto para os helmintos. Entre os adultos a faixa etária de 26 a 50 anos foi a que teve maior frequência de indivíduos parasitados $(15 \%, \mathrm{n}=11)$.

Ascaris lumbricoides foi mais frequente entre as crianças de 1 a 3 anos (33\%, $\mathrm{n}=8)$ e 4 e $6 \operatorname{anos}(30 \%, n=4)$. Entre as crianças menores de 1 ano, observou-se $3 \%$ de prevalência $(\mathrm{n}=5)$ para esse parasita. Giardia lamblia foi o protozoário mais frequente aparecendo em todas as categorias e em todas as faixas de idade. As maiores frequências foram observadas entre as crianças de 1 a 3 anos $(n=39), 4$ a 6 anos $(n=32)$ e 7 a 9 anos (n=34) (Tabela 2).

Tabela 2 - Distribuição dos parasitas por espécie e faixa etária (AL: Ascaris lumbricoides, TT: Trichuris trichiura, ANC: Ancilostomídeos, SS: Strongiloides stercoralis, EH: Entamoeba histolytica, GL: Giardia lamblia)

\begin{tabular}{l|c|c|c|c|c|c|c|c|c} 
& \multicolumn{4}{c}{ Helmintos } & \multicolumn{3}{c}{ Protozoários } \\
\hline Categoria & Faixa etária & AL & TT & ANC. & SS & EH & GL & N & \% \\
\hline \multirow{5}{*}{ Crianças } & $<1$ ano & 0 & 1 & 1 & 1 & 0 & 1 & 4 & 2 \\
\cline { 2 - 11 } & 1 a 3 anos & $\mathbf{8}$ & 0 & 0 & 0 & 0 & $\mathbf{3 9}$ & $\mathbf{4 7}$ & $\mathbf{2 2}$ \\
\cline { 2 - 11 } & 4 a 6 anos & $\mathbf{4}$ & 0 & 1 & 0 & 5 & $\mathbf{3 2}$ & $\mathbf{4 2}$ & $\mathbf{1 9}$ \\
\cline { 2 - 11 } & 7 a 9 anos & 3 & 0 & 0 & 2 & 5 & $\mathbf{2 4}$ & $\mathbf{3 4}$ & $\mathbf{1 6}$ \\
\cline { 2 - 11 } & 10 a 12 anos & 0 & 0 & 0 & 0 & 6 & 9 & 15 & 7 \\
\hline Adolescentes & 13 a 17 anos & 2 & 0 & 1 & 0 & 2 & 10 & 15 & 7 \\
\hline \multirow{4}{*}{ Adultos } & 18 a 25 anos & 1 & 0 & 0 & 0 & 1 & $\mathbf{2 1}$ & $\mathbf{2 3}$ & 11 \\
\cline { 2 - 11 } & 26 a 50 anos & 1 & 0 & 0 & 0 & 3 & $\mathbf{2 3}$ & $\mathbf{2 7}$ & 12 \\
\cline { 2 - 10 } & $>50$ anos & $\mathbf{5}$ & 0 & 0 & 0 & 1 & 5 & 11 & $\mathbf{1 5}$ \\
\cline { 2 - 10 } & TOTAL & $\mathbf{2 4}$ & $\mathbf{1}$ & $\mathbf{3}$ & $\mathbf{3}$ & $\mathbf{2 3}$ & $\mathbf{1 6 4}$ & $\mathbf{2 1 8}$ & $\mathbf{1 0 0}$ \\
\hline
\end{tabular}

Nos últimos anos, o cenário das parasitoses intestinais na América Latina tem sido pouco alterado. Sua elevada prevalência e diversidade de manifestações clínicas representam um problema de Saúde Pública, especialmente nos países menos desenvolvidos. As enfermidades intestinais mais importantes são as provocadas por protozoários e/ou helmintos, cuja transmissão ocorre pela ingestão de formas parasitárias como ovos, larvas, cistos ou oocistos (FREITAS et al., 2005).

O parasitismo intestinal acomete grande parte da população em situações sócios econômicas difíceis, e em cidades pouco desenvolvidas, nas quais ainda é realidade comum a falta de saneamento básico e água tratada. É o que defendem Santos et al. (1999) ao afirmarem que a alta positividade de enteroparasitismo ocorre nos bairros mais carentes do município de Feira de Santana-BA em decorrência não só da 


\section{Ensino, Saúde e Ambiente - V 7 (1), Edição Especial, maio de 2014}

questão social, mas, principalmente, pela falta de infraestrutura relativa ao saneamento básico, tanto na zona rural como nas zonas suburbanas e bairros periféricos.

Costa-Macêdo et al. (1999), mostram o alto grau de parasitismo por Ascaris lumbricoides com infecções a partir de um ano e o aumento da prevalência de acordo com o aumento da idade. Esse mesmo padrão de distribuição foi observado por Ludwig et al. (1999) ao analisarem a frequência de parasitoses em várias comunidades em Assis-SP: Giardia lamblia e Ascaris lumbricoides como parasitas mais frequentes em diferentes faixas etárias, principalmente entre crianças na faixa etária de 3 a 6 anos, com aumento progressivo na frequência até os seis anos de idade, quando começa a decrescer. Uma conclusão importante dessa pesquisa mostra que no período analisado (3 anos) houve uma tendência para queda na prevalência das parasitoses intestinais com o aumento das ligações de água e esgoto em todas as regiões da cidade de Assis, mostrando uma relação direta entre a melhoria das condições de saneamento básico e o decréscimo de casos de enteroparasitismo.

O estudo realizado por Uchôa et al. (2001) também constatou a maior prevalência de Giardia lamblia e Ascaris lumbricoides entre as crianças de 0 a 6 anos atendidas por creches comunitárias do Rio de Janeiro. Neste estudo a alta frequência encontrada para estes parasitas pode ser relacionada a inexistência de estrutura de saneamento básico e a dificuldade de abastecimento de água potável no município

De acordo com Costa-Macedo et al. (1999) no Brasil, o parasitismo intestinal nos dois primeiros anos de vida é menos conhecido que em outras faixas etárias, e pode estar relacionado ao período do desmame da criança, quando ocorre a introdução de novos alimentos e inicia-se uma etapa do desenvolvimento que lhe permite maior mobilidade no ambiente. De qualquer forma, O parasitismo de menores de 24 meses pode (e deve) estar refletindo o elevado grau de contaminação dos ambientes peri e domiciliar nesse local, resultante das precárias condições de saneamento básico existentes. Os níveis crescentes da prevalência por idade observados nas crianças devem estar relacionados ao processo de crescimento e desenvolvimento infantil (mobilidade e interação com o ambiente) e ao maior tempo de exposição às condições ambientais.

Vale ressaltar que o parasitismo por Ascaris lumbricoides em menores de dois anos é um fator preocupante, por que é nesse grupo que as complicações decorrentes do parasitismo são mais graves. Sangkhathat et al. (2003) descrevem dois casos graves envolvendo hemorragia gastrointestinal, perfuração do intestino e obstrução intestinal, 


\section{Ensino, Saúde e Ambiente - V 7 (1), Edição Especial, maio de 2014}

esta última provocada pela presença do enovelamento de vermes no intestino. Ambos os casos estão relacionados a quadros de desnutrição e anemia.

Valentine et al. (2001) descrevem a ocorrência de pneumonia em criança de dois anos em decorrência de síndrome de Löefler, ou seja, o problema respiratório foi causado pela passagem ou retenção das larvas de Ascaris lumbricoides no trato respiratório.

Araújo e Guimarães (2000) reportam um caso de localização ectópica de Ascaris lumbricoides em um paciente de um ano de idade. Os autores relatam ainda que a criança, atendida no hospital de Sete Lagoas-MG, era vítima de desnutrição acentuada e apresentou episódio súbito de vômitos aquosos, com grande quantidade de vermes adultos de Ascaris lumbricoides. Verificou-se que apresentava também conjuntivite, com produção de secreção purulenta e problemas na drenagem das lágrimas. $\mathrm{O}$ exame oftalmológico mostrou a presença de um verme parcialmente exteriorizado no canal lacrimal. Os autores referem-se a denominação de "áscaris errático" ao verme que se localiza em habitat anormal, como no apêndice cecal (causando apendicite aguda), canal colédoco (causando sintomas hepatobiliares), canal de Wirsung (causando pancreatite aguda), trompa de Eustáquio (causando otites), intra hepático (causando abcesso no fígado), rim e uretra (pielonefrite e hidronefrose) e cérebro (causando abcesso cerebral).

Como pode ser observado as enteroparasitoses se apresentam como grave problema de saúde pública, principalmente quando consideramos que as consequências envolvem o baixo rendimento escolar entre as crianças e a incapacitação para o trabalho entre os adultos (NEVES, 2011)

No Brasil o problema é mais sério, uma vez que lamentavelmente há falta de políticas de educação sanitária profundas e sérias. A erradicação desses parasitas requer melhorias das condições socioeconômicas, no saneamento básico e na educação sanitária, além de mudanças de certos hábitos culturais (TAVARES-DIAS e GRANDINI, 1999).

Dessa forma, enquanto políticas mais abrangentes no âmbito da saúde e da educação não são colocadas em prática, tomamos a iniciativa de discutir o problema das enteroparasitoses no município de Nova Esperança do Piriá. Para isso, elaboramos atividades educativas que envolveram professores e alunos de uma escola municipal.

As atividades foram realizadas na Escola Municipal de Ensino Fundamental Maria Laurita envolvendo professores e alunos de sexto ano do Ensino Fundamental e culminou com a III Feira de Ciências da Escola.

Edição Especial com os melhores trabalhos apresentados no IV ENECiências: UFF - 13 a 16 de maio de 2014. 


\section{Ensino, Saúde e Ambiente - V 7 (1), Edição Especial, maio de 2014}

No primeiro momento da atividade apresentamos aos professores e alunos a ideia de trabalharmos o tema das parasitoses intestinais. Em seguida foram todos encaminhados à sala de informática da escola com o objetivo de levantar mais informações sobre o tema e sua problemática. A pesquisa também foi realizada nos livros didáticos disponíveis na biblioteca.

No segundo momento, em novo encontro com a turma, fizemos a socialização através de debates, sobre o material pesquisado. Nesse momento apresentamos o levantamento realizado em Nova Esperança do Piriá. Em seguida, montamos uma roda de conversa para discutir esses dados e propor ações.

No terceiro encontro, com os alunos já informados pelos professores responsáveis que iriam trabalhar essa temática na feira de ciências da escola, separamos quatro grupos com seis alunos: $\mathrm{O}$ primeiro grupo discutiu os problemas causados pelo parasitismo de Giardia lamblia (giardíase), o segundo grupo, discutiu Entamoeba histolytica (amebíase). O teceiro grupo ficou responsável por discutir as doenças relacionadas a Ascaris lumbricoides (ascaridíase), Strongiloides stercoralis (estrongiloidíase) e Ancilostomídeos (ancilostomíase). A última equipe ficou responsável pelo Trichuris trichiura (tricuríase).

Os trabalhos foram apresentados pelos alunos na III Feira de Ciências da escola através de cartazes, folders que abordavam as características das doenças, principais sintomas, mecanismos de contágio e medidas profiláticas. Também foram apresentados os dados levantados em Nova Esperança do Piriá, uma vez que além de alunos e professores de outras turmas, o evento recebeu a visita de funcionários da escola e da comunidade como um todo.

Com a execução dessa atividade acreditamos que assumimos uma posição ativa no que tange a prevenção e promoção da saúde no município. Logicamente que a solução desse problema necessita de muitas outras ações efetivas e integradas que envolvam além das escolas, as secretarias de saúde e educação, bem como a comunidade como um todo.

Entendemos com isso que a educação para saúde é uma ferramenta imprescindível para auxiliar o processo de sensibilização do indivíduo acerca dos benefícios advindos dos bons hábitos de higiene, além de influenciar diretamente na preservação da saúde coletiva

Toscani et al. (2007) defendem que a Educação em Saúde no controle das parasitoses intestinais tem se mostrado uma estratégia de baixo custo capaz de atingir Edição Especial com os melhores trabalhos apresentados no IV ENECiências: UFF - 13 a 16 de maio de 2014. 


\section{Ensino, Saúde e Ambiente - V 7 (1), Edição Especial, maio de 2014}

resultados significativos e duradouros. Para as crianças especialmente, ajuda a desenvolver a responsabilidade perante o seu próprio bem estar, a prática de hábitos saudáveis, além de contribuir para a manutenção de um ambiente são.

No entanto, para que isso ocorra, é importante que o processo educativo não se dê de maneira impositiva, mas em um ambiente prazeroso, propiciando uma relação direta entre os conteúdos e o dia-a-dia. Foi isso que pretendemos fazer ao propor essa ação.

\section{CONSIDERAÇÕES FINAIS}

A maioria das infecções parasitárias é adquirida através da transmissão fecaloral causada pela ingestão de água e alimentos contaminados em decorrência de inadequada infraestrutura de saneamento. São fatores determinantes para o elevado índice de pessoas parasitadas baixa renda familiar, famílias numerosas, péssimas condições de higiene do ambiente, domicílio e pessoal, além da falta de conhecimento sobre a profilaxia das doenças parasitárias.

De acordo com Melo et al. (2004) as infecções intestinais parasitárias ocorrem por um desequilíbrio ecológico entre parasita-hospedeiro-ambiente. A melhoria das condições de vida através do saneamento adequado, combate à desnutrição e do desmame precoce, educação para a prevenção, acesso universal ao sistema de saúde, são medidas que efetivamente diminuiriam a infecção parasitária.

Verificamos que a população de Nova Esperança do Piriá é acometida por parasitas intestinais desde a infância e nossa experiência como professoras da educação infantil nos mostra o quanto essa problemática está longe de ser discutida em nossas escolas e em nossa comunidade.

Neves (2011) defende que o conhecimento sobre a sequência de eventos que acontecem no desenvolvimento de uma doença tem aplicações práticas, não só no emprego de terapêuticas especificas como também na definição dos métodos de prevenção e controle. Nesse contexto, medidas de prevenção primárias, ou seja, aquelas que procuram impedir que o indivíduo adoeça, exigem muitas vezes que medidas muito elementares sejam implantadas para controlar os fatores de risco. No entanto, mesmo que básicas essas medidas ainda estão muito longe da realidade da maioria das cidades brasileiras: moradia adequada, saneamento ambiental (incluindo tratamento de água, sistemas de esgoto, coleta de lixo) educação, alimentação adequada, lazer, etc. 
Ensino, Saúde e Ambiente - V 7 (1), Edição Especial, maio de 2014

Cabe ainda destacar que apesar do êxito dessa atividade os resultados de nossa pesquisa demonstram a necessidade da implementação de medidas de saneamento básico e programas de saúde contínuos visando a educação sanitária. A associação destas medidas possibilitaria uma melhoria da condição de vida da comunidade e consequentemente, reduziria o número de pessoas parasitadas, o que para as crianças implicaria em um melhor desenvolvimento e rendimento escolar.

\section{REFERÊNCIAS BIBLIOGRÁFICAS}

ARAÚJO, E. H. P.; GUIMARÃES, S. S. Obstrução naso lacrimal em criança: Ascaris lumbricoides como uma causa incomum. Arq. Bras. Oftalmol. 63(5), outubro/2000.

COSTA-MACEDO, L. M.; COSTA, M. C. E.; ALMEIDA, L. M.. Parasitismo por Ascaris lumbricoides em crianças menores de dois anos: estudo populacional em comunidade do Estado do Rio de Janeiro. Cad. Saúde Pública, Rio de Janeiro, 15(1):173-178, jan-mar, 1999.

FREITAS, A. A. Organização Mundial de La Salud. Comite de Expertos en Prevenciòn y Control de Infecciones Parasitarias Intestinales. Ginebra: OMS. 2005

LUDWIG, K. M.; FREI, F.; FILHO, F.; ALVARES; RIBEIRO-PAES, J. T. Correlação entre condições de saneamento básico e parasitoses intestinais na população de Assis, Estado de São Paulo. Revista da Sociedade Brasileira de Medicina Tropical. 32(5):547-555, set-out, 1999.

MACEDO, H. S. Prevalência de Parasitos Comensais e Intestinais em crianças de Escola da Rede Pública Municipal de Paracatu (MG). RBAC, p. 209-213. 2005.

MELO, M. C. B.; KLEM, V. G. Q.; MOTA, J. A. C.; PENNA, F. J. Parasitoses Intestinais. Rev Med Minas Gerais; 14 (1 Supl. 1): S3-S12. 2004.

NEVES, David Pereira. Parasitologia Humana. 13 ${ }^{\mathrm{a}}$ ed. Atheneu, São Paulo. 2011.

SANGKHATHAT, S.; PATRAPINYOKUL, S.; WUDHISUTHIMETHAWEE, P.; CHEDPHAOPAN, J.; MITAMUN, W. Massive Gastrointestinal Bleeding in Infants with Ascariasis. Journal of Pediatric Surgery, Vol 38, No 11 (November). 2003.

SANTOS, J. F.; CORREIA, J. E.; GOMES, S. S. B. S.; SILVA, P. C.; BORGES, F. A. Estudo das Parasitoses Intestinais na Comunidade Carente dos Bairros Periféricos do Município de Feira de Santana (BA), 1993 - 1997. Sitientibus, Feira de Santana, n.20, p.55-67, jan./jun. 1999.

SENNA-NUNES, M. S.; Mello-Silva, C. C; Barbosa, G. L. S.; Dias, B.; Lorca, F.O.; Simões, L. G. Ações Educativas para a Prevenção de Parasitoses aplicadas em Escolas no Município de Nova-Iguaçu, Rio de Janeiro, Brasil. XV Congresso LatinoAmericano de Parasitologia, São Paulo. 2001.

TAVARES-DIAS, M.; GRANDINI, A. A.. Prevalência e aspectos epidemiológicos de enteroparasitoses na população de São José da Bela Vista, São Paulo. Revista da Sociedade Brasileira de Medicina Tropical. 32(1):63-65, jan-fev, 1999.

TOSCANI, N. V.; SANTOS, A. J. D. S.; SILVA, L. L. M.; TONIAL, C. T.; CHAZAN, M.; WIEBBELLING, A. M. P.; MEZZARI, A. Desenvolvimento e análise de jogo Edição Especial com os melhores trabalhos apresentados no IV ENECiências: UFF - 13 a 16 de maio de 2014.

ISSN 1983-7011 
educativo para crianças visando à prevenção de doenças parasitológicas. Interface Comunicação e Saúde, v.11, n.22, p.281-94, mai/ago 2007.

UCHÔA, C. M. A.; LOBO, A. G. B.; BASTOS, O. M. P.; MATOS, A. D. Parasitoses intestinais: prevalência em creches comunitárias da cidade de Niterói, Rio de Janeiro Brasil. Rev. Inst.Adolfo Lutz, 60(2): 97-101. 2001.

VALENTINE, C. C.; HOFFNER, R. J.; HENDERSON, S. O. Three Common Presentations of Ascariasis Infection in an Urban Emergency Department. The Journal of Emergency Medicine, Vol. 20, No. 2, pp. 135-139, 2001. 